Introduction: The neurotransmitters serotonin and dopamine are implicated in normal cognitive functioning. In healthy volunteers, dopamine depletion produces selective deficits of spatial working memory (SWM) while serotonin depletion results in deficits on a delayed word recall task (delayed verbal learning). Serotonin 5-HT1A receptors in man are found in postsynaptic locations in hippocampus and neocortex, and as somatodendritic autoreceptors in the raphe nuclei. 5-HT1A agonists have been shown to preferentially increase dopamine in the prefrontal cortex (PFC) acting by means of the 5-HT1A autoreceptors, which may influence cognitive function and memory in particular. We aimed to investigate the relationship between serotonin activity and memory performance in healthy volunteers.

Method: Ten male and 10 female healthy volunteers (aged between 18 and 60 years) were tested in a randomized, double-blind, crossover design receiving both buspirone $(20 \mathrm{mg})$ and placebo over the course of the study. All subjects completed tests of reaction time, SWM and verbal learning.

Results: Repeated-measures ANOVA showed significant decrement in delayed word recall performance after buspirone administration. SWM and RT data will be available for discussion at the time of presentation. Conclusions: Single dose of buspirone caused slight but nonetheless significant worsening in delayed verbal recall in healthy volunteers. This outcome may be because of 1) verbal memory decrements as a result of nondirect 5-HT1A receptor action on dopamine function in the PFC or, alternatively, 2) buspironeinduced changes in serotonergic function in the hippocampus.

\section{Altered neurophysiological parameters in an animal model of hyperactivity}

\section{TL Pitcher, JR Wickens, JNJ Reynolds}

University of Otago, New Zealand

Background: The spontaneously hypertensive rat (SHR) is commonly used as an animal model of attention deficit hyperactivity disorder-like behavioural characteristics. Little is known about the physiology of the SHR and its genetic control, Wistar-Kyoto (WKY). The aim of this research was to investigate the physiology of neurons in a brain area involved in movement initiation, the striatum.

Methods: Male rats from the Wistar (WI), SHR and WKY strains were anaesthetized with urethane $(1.8-2.4 \mathrm{~g} / \mathrm{kg})$. In vivo intracellular recordings were obtained using sharp microelectrodes from spiny projection neurons in the dorsal striatum. Cellular proper- ties were measured from electrophysiological records of spontaneous activity and neuronal responses to intracellular current injection.

Results: Recordings were made from 71 striatal spiny projection neurons (WI, $n=24$; SHR, $n=24$; WKY, $n=23$ ). Most cellular properties were similar across the three rat strains. However, two parameters of action potential firing differed between the SHR and WKY strains. (Current evoked action potential amplitude: SHR $76.5 \pm 7.5 \mathrm{mV}$, WKY $70.1 \pm 8.7 \mathrm{mV}$; duration: SHR $0.60 \pm 0.09 \mathrm{~ms}$, WKY $0.67 \pm 0.06 \mathrm{~ms} ; P<0.05$ one-way ANOVA.) The cellular properties of the WI strain were intermediate and not significantly different to either SHR or WKY.

Conclusions: The action potential parameter differences between the SHR and WKY strains may be related to the behavioural differences between the two strains. At the cellular level, this difference may also indicate that the two strains contain differences in membrane conductances in the striatal spiny projection neurons.

\section{Depression and health risk factors in the North West Adelaide Health Study \\ N Potts', D Wilson'2, A Taylor ${ }^{3}$, T Gill', G Schrader ${ }^{1,4}$, R Ruffin ${ }^{2}$}

'University of Adelaide; ${ }^{2}$ Health Observatory, Department of Medicine, The Queen

Elizabeth Hospital; 3 Population Research and Outcome Studies Unit,

South Australian Department of Health; and ${ }^{4}$ Department of Psychiatry,

The Queen Elizabeth Hospital, Adelaide, Australia

Background: The North West Adelaide Health Study is a population-based biomedical cohort study investigating the prevalence of chronic conditions and health-related risk factors. This study is based on a randomly selected group of individuals over the age of 18 , who agreed to be involved in a longitudinal health study.

Methods: Over the past 18 months, 3488 participants were assessed. General demographic and socioeconomic data were collected. Participants were assessed for the presence of a number of chronic conditions including major depression, chronic lung disease, quality of life using the SF-36 and health risk factors such as body mass index, smoking and exercise.

Results: Of the 3488 participants, $12.5 \%$ (436/3488) met criteria for depression. Depression was statistically significantly more likely among those who were current smokers, obese, had a high waist-hip ratio or waist circumference than those without these risk factors but less likely in those who undertook exercise. Depression was also associated with significantly lower scores on all the quality of life scores as 